\title{
Full Paper \\ Prevention of febrile neutropenia: use of granulocyte colony-stimulating factors
}

\author{
S Kelly*,I and D Wheatley ${ }^{2}$ \\ 'Plymouth Oncology Centre, Derriford Hospital, Plymouth PL6 8DH, UK; ${ }^{2}$ Oncology Centre, Royal Cornwall Hospital, Truro, Cornwall TRI 3LJ, UK
}

\begin{abstract}
There is good evidence to suggest that dose intensity is important when considering the effectiveness of adjuvant chemotherapy in patients with breast cancer. However, the development of chemotherapy-induced febrile neutropenia can lead to reduction in dose intensity and other treatment modifications, which may negatively affect patient outcomes. Febrile neutropenia can be prevented by the use of primary prophylactic treatment, notably with granulocyte colony-stimulating factors. This practice is supported by international guidelines, all of which recommend that primary prophylaxis with granulocyte colony-stimulating factors should be used with chemotherapy where the risk of febrile neutropenia is $20 \%$ or greater. British Journal of Cancer (2009) I 0I, S6-SI 0. doi: I 0.1038/sj.bjc.6605269 www.bjcancer.com
\end{abstract} (c) 2009 Cancer Research UK

Keywords: dose intensity; febrile neutropenia; G-CSF; guidelines; prophylaxis

Use of a chemotherapy regimen containing a taxane or an anthracycline in the adjuvant treatment of patients with breast cancer has been shown to have benefits in terms of both time to disease progression and overall survival (Martin et al, 2005; Peto et al, 2007). However, such regimens are also associated with the potentially serious side effects of febrile neutropenia (FN) and neutropenic sepsis (Martin et al, 2005; Peto et al, 2007). FN is not only a major risk factor for morbidity and mortality in patients with cancer (Aapro et al, 2006; Herbst et al, 2009), but its development can also lead to a decision to reduce the chemotherapy dose and delay subsequent treatment cycles (Aapro et al, 2006). Such treatment modifications are a particular concern when chemotherapy is given with curative intent (Aapro et al, 2006), and hence the importance of FN prevention.

FN can be prevented through the prophylactic use of haematopoietic cell growth factors (e.g., granulocyte colony-stimulating factors, G-CSF) - a strategy supported by current guidelines for patients deemed to be at high risk of FN (Aapro et al, 2006; Smith et al, 2006; Segal et al, 2008). The indications for prophylactic administration of G-CSF are based on various risk factors, including the degree of myelosuppression associated with the chemotherapy regimen, and specific patient characteristics (Aapro et al, 2006; Smith et al, 2006).

The management of serious neutropenic events is described elsewhere in this supplement (Cameron, 2009), as is the antibioticbased prophylaxis of FN (Cullen and Baijal, 2009). This article focuses on the potential consequences of modifying chemotherapy dose density in response to $\mathrm{FN}$, and the alternative approach primary prevention of FN through the prophylactic use of G-CSF.

\section{FN MORBIDITY AND MORTALITY}

Chemotherapy-induced neutropenia is a major risk factor for infection-related morbidity (Aapro et al, 2006), including fungal infections, Gram-negative sepsis, pneumonia and other lung

*Correspondence: Professor S Kelly; E-mail: Steve.Kelly@phnt.swest.nhs.uk disease, cerebrovascular disease and disorders of the liver and kidney (Kuderer et al, 2006).

The mortality rates associated with FN range from 2 to $21 \%$ (Herbst et al, 2009), and the risk of death is increased by various factors, including patient characteristics, type of malignancy, presence of comorbidities and infectious complications (Kuderer et al, 2006).

\section{DOSE INTENSITY}

Development of FN and neutropenic sepsis leads to significant morbidity and mortality, and is commonly regarded as an indication for a dose reduction or cycle delay among patients receiving chemotherapy (Aapro et al, 2006). However, there is evidence that such changes to chemotherapy dose intensity (DI, dose delivered divided by the overall duration of treatment) can have a negative effect on treatment outcomes (Wood et al, 1994; Bonadonna et al, 1995; Leonard et al, 2003; Bonneterre et al, 2005; Chirivella et al, 2006).

A retrospective analysis of patients undergoing adjuvant treatment with classical 28-day CMF (cyclophosphamide, methotrexate and fluorouracil) showed a major survival benefit among those who received $85 \%$ or more of the intended dose (Bonadonna et al, 1995). Delivery of $65-85 \%$ of the planned dose was associated with some benefit, but patients who received less than $65 \%$ fared no better than did those who received no chemotherapy at all.

Another study compared three regimens of FAC (fluorouracil, doxorubicin and cyclophosphamide) that differed in their DI moderate-DI FAC (fluorouracil $40 \mathrm{mg} \mathrm{m}^{-2}$ on days 1 and 8 , and doxorubicin $40 \mathrm{mg} \mathrm{m}^{-2}$ and cyclophosphamide $400 \mathrm{mg} \mathrm{m}^{-2}$ on day 1 for six cycles), higher-DI FAC (the same total dose as moderateDI FAC but delivered in just four cycles) and lower-DI FAC (half the total dose of high-DI and moderate-DI FAC, delivered in four cycles) (Wood et al, 1994). After 3 years, there was no difference between the moderate-DI and higher-DI groups in terms of 
disease-free and overall survival, but both outcomes were significantly poorer in the lower-DI group. The authors propose a threshold effect for chemotherapy delivery, that is, a DI below which the benefit is significantly reduced (Wood et al, 1994).

An association between increased DI and improved outcomes has also been reported by the French Adjuvant Study Group (Bonneterre et al, 2005), which compared two regimens of FEC. Patients receiving adjuvant chemotherapy for breast cancer were given either FEC 50 (fluorouracil $500 \mathrm{mg} \mathrm{m}^{-2}$, epirubicin $50 \mathrm{mg} \mathrm{m}^{-2}$ and cyclophosphamide $500 \mathrm{mg} \mathrm{m}^{-2}$ ) or FEC 100 (as FEC 50 but with the epirubicin dose increased to $100 \mathrm{mg} \mathrm{m}^{-2}$ ). At 10 years, there was a significant benefit in favour of FEC 100 in the rates of disease-free survival (FEC 50, 45.3\% vs FEC 100, 50.7\%, $P=0.036)$ and overall survival (50.0 vs $54.8 \%$, respectively, $P=0.038)$

Looking specifically at the reduced DI brought about by dose delay, a retrospective analysis of the records of 793 patients who received adjuvant chemotherapy for breast cancer between 1980 and 2000 has shown that, after a median follow-up of 10 years, patients exposed to more than two cycle delays had poorer eventfree and overall survival, compared with those whose treatment was delivered on time (Chirivella et al, 2006). In this study, treatment delay was defined as any cycle being delivered more than 3 days later than planned, the whole course being completed more than 15 days later than planned or a delivery of less than $95 \%$ of the planned DI.

The association between the development of severe neutropenia and reduced DI has been investigated in an audit of 422 patients with breast cancer who received adjuvant chemotherapy (mainly CMF or anthracycline-based) in 15 UK centres; $29 \%$ of the patients had at least one neutropenic event (defined as hospitalisation because of FN, a dose delay of 7 days or more because of neutropenia, and/or a dose reduction of $15 \%$ or more because of neutropenia), and $17 \%$ of the patients received less than $85 \%$ of the planned total dose of their regimen (Leonard et al, 2003). Patients who experienced a neutropenic event received a significantly lower DI than those who did not. Around $40 \%$ of patients undergoing CMF-based chemotherapy and $32 \%$ of patients undergoing anthracycline-based chemotherapy who experienced a neutropenic event received less than $85 \%$ of their intended dose. Interestingly, only $5.2 \%$ of patients in the study received haematopoietic cell growth factors at any time during their treatment.

\section{CHOICE OF CHEMOTHERAPY}

There are many factors to consider when choosing a suitable chemotherapy regimen for an individual patient, and little research to show why some patients who seem suitable for a particular regimen do not receive it. However, there are anecdotal reports either that patients may decline effective chemotherapy or that physicians may be unwilling to prescribe it, because of concerns about toxicity - even when the likely side effects can be effectively prevented or managed. For example, the findings of a survey of 50 UK oncologists, conducted in 2008, suggested that FN was a deterrent to the prescription of docetaxel, doxorubicin and cyclophosphamide (TAC) for patients with operable node-positive early-stage breast cancer. Only $58 \%$ of eligible patients were considered for the regimen, and only $32 \%$ of the eligible population actually received it (Oncologist Breast Cancer Study, 2008). The respondents cited concern about FN as the major barrier to prescribing TAC. Only $24 \%$ of the oncologists used G-CSF for primary prophylaxis of FN.

Gounaris et al (2008) have shown that a patient's age can also be a major factor affecting the decision to use particular chemotherapy regimens. In their study, the mean ages of patients with node-positive breast cancer prescribed taxane-based chemotherapy, anthracycline-based chemotherapy or no chemotherapy were 52.7, 59.4 and 73.2 years, respectively. Whether age per se should affect the choice of chemotherapy will be discussed in detail later.

\section{GUIDELINES ON PREVENTION OF FN}

Chemotherapy dose reduction/delay is not the only strategy available for reducing FN-related morbidity and mortality. Another option is FN prevention through prophylactic treatment with G-CSF, granulocyte-macrophage colony-stimulating factors (GM-CSF) and/or antibiotics (Herbst et al, 2009).

Haematopoietic cell growth factors stimulate the proliferation and survival of neutrophils and their precursors, and thereby reduce the severity and duration of chemotherapy-induced neutropenia and FN (Ozer et al, 2000; Komrokji and Lyman, 2004; Segal et al, 2008).

Indications for the primary prevention of FN through the use of G-CSFs for patients undergoing chemotherapy have been issued by three well-respected oncology organisations - the American Society of Clinical Oncology (Smith et al, 2006), the European Organization for Research and Treatment of Cancer (Aapro et al, 2006) and the National Comprehensive Cancer Network (Segal et al, 2008). They all broadly agree that any patient with an FN risk greater than $20 \%$ should receive primary prophylaxis with G-CSF with each cycle of chemotherapy (Figure 1). In some instances, the chemotherapy regimen itself carries an FN risk that exceeds this threshold (Aapro et al, 2006). If the FN risk associated with the regimen is $10-20 \%$, the physician should consider whether patient factors such as age, advanced disease or comorbidities take the overall risk beyond $20 \%$. If the chemotherapy regimen is considered to present an FN risk of less than 10\%, primary prophylaxis with G-CSF should not be offered routinely - unless there is thought to be a huge risk of serious FN complications, such as death. However, it is highly likely that G-CSF primary prophylaxis is not always used routinely in clinical practice, despite these clear, evidence-based guidelines.

\section{EVIDENCE SUPPORTING PRIMARY PROPHYLAXIS OF FN}

In a meta-analysis of 17 randomised controlled trials involving more than 3400 patients undergoing chemotherapy, infectionrelated mortality was $1.5 \%$ in those who received primary prophylaxis with G-CSF, compared with $2.8 \%$ in controls (relative risk $(\mathrm{RR})=0.055) \quad$ (Kuderer et al, 2007). The rate of early morbidity fell from $3.4 \%$ in controls to $2.7 \%$ in patients receiving G-CSF. FN occurred once or more in $39.5 \%$ of controls and $22.4 \%$ of G-CSF-treated patients $(R R=0.54)$. In 10 trials that monitored DI, the meta-analysis found that all G-CSF recipients had a DI higher than $90 \%$. By contrast, DI in the control arms was lower than $85 \%$ in six of the 10 trials.

The efficacy of pegfilgrastim, a prolonged-action pegylated form of G-CSF, in the prevention of FN associated with chemotherapy based on docetaxel $\left(100 \mathrm{mg} \mathrm{m}^{-2}\right)$ in breast cancer was assessed in a study of 928 patients with metastatic breast cancer randomised to either placebo or pegfilgrastim (Vogel et al, 2005). Compared with the placebo group, the pegfilgrastim-treated patients had a significantly lower rate of FN (17 vs $1 \%$, respectively, $P<0.001$ ), FN-related hospital admissions $(14$ vs $1 \%, P<0.001)$ and use of intravenous antibiotics for the management of infections $(10 \mathrm{vs}$ $2 \%, P<0.001)$. During cycle 1 of chemotherapy, $11 \%$ of patients who received placebo developed FN (i.e., two-thirds of all episodes of FN in the placebo group), compared with $1 \%$ of pegfilgrastim recipients. As patients who were given placebo and developed FN were then allowed to receive pegfilgrastim during subsequent chemotherapy cycles, the DI was not significantly different between the two groups. 


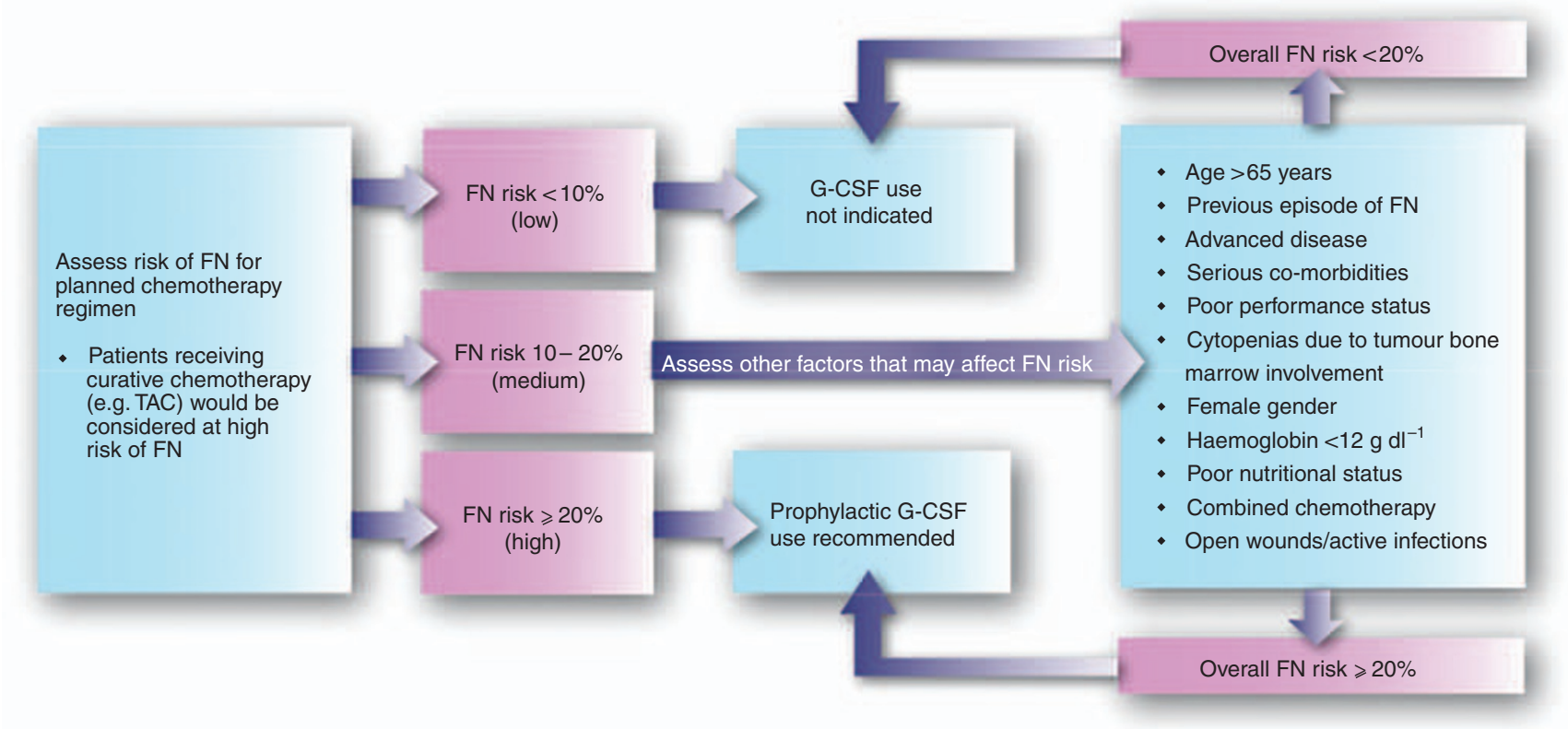

Figure I Algorithm for FN prevention in patients receiving chemotherapy, based on guidelines by the European Organization for Research and Treatment of Cancer (Aapro et al, 2006) and the American Society of Clinical Oncology (Smith et al, 2006).

The international guidelines are supported further by recent results from the GeparTrio trial, in which patients with breast cancer who received neoadjuvant TAC were given the following prophylactic treatments: the antibiotic ciprofloxacin $(500 \mathrm{mg}$ twice daily on days 5-14), non-pegylated G-CSF $\left(5 \mu \mathrm{g} \mathrm{kg}^{-1} \mathrm{day}^{-1}\right.$ filgrastim or $150 \mu \mathrm{g} \mathrm{m}^{-2}$ day $^{-1}$ lenograstim on days 5-10), pegfilgrastim (6 $\mathrm{mg}$ on day 2$)$ or a combination of the pegfilgrastim and the ciprofloxacin schedules (von Minckwitz et al, 2008). Both FN and grade 4 neutropenia were significantly more frequent in patients who received either ciprofloxacin alone or non-pegylated G-CSF, compared with those given pegfilgrastim with or without ciprofloxacin. Indeed, no patients receiving the combination of pegfilgrastim and ciprofloxacin developed FN during chemotherapy cycle 1 .

The combined approach also proved beneficial in a study by Gounaris et al (2008). In the study, patients given the complete primary prophylaxis schedule of lenograstim $(263 \mu \mathrm{g}$ subcutaneously on days 4-10) and ciprofloxacin (500 mg twice daily on days $5-14$ ) had an FN rate of 0.8 , and $7 \%$ needed a modification to their chemotherapy regimen (Gounaris et al, 2008). By contrast, when prophylaxis was inadequate (usually because G-CSF was started later than day 5), the risk of FN was 15 , and $36 \%$ of patients required modification to their chemotherapy.

\section{FN PROPHYLAXIS IN CLINICAL PRACTICE}

Physicians need to be aware of the FN risk associated with each regimen that they prescribe and take into account the patientrelated factors that increase that risk. Primary prophylaxis with G-CSF should always be offered when the risk of FN is $20 \%$ or greater. Prophylactic use of antibiotics should also be considered, as discussed elsewhere in this supplement by Cullen and Baijal.

G-CSF treatment should start within $24-72 \mathrm{~h}$ of the chemotherapy dose, and be administered for long enough to allow adequate neutrophil recovery. Treatment schedules associated with three commonly used G-CSF formulations are summarised in Table 1. Pegfilgrastim is administered just once per cycle, whereas shorter acting products are used daily. The duration of use of daily G-CSF
Table I G-CSF treatment schedules

\begin{tabular}{|c|c|c|c|c|}
\hline Formulation & Route & Dose & Frequency & Duration \\
\hline Lenograstim & Subcutaneous & $150 \mu \mathrm{g} \mathrm{m}^{-2}$ & Daily & $\begin{array}{l}28 \text { consecutive } \\
\text { days }\end{array}$ \\
\hline Filgrastim & Subcutaneous & $5 \mu \mathrm{g} \mathrm{kg}^{-1}$ & Daily & Up to 38 days \\
\hline Pegfilgrastim & Subcutaneous & $6 \mathrm{mg}^{-1}$ & $\begin{array}{l}\text { Once per } \\
\text { cycle }\end{array}$ & Once per cycle \\
\hline
\end{tabular}

is variable. Trial evidence has shown that $\mathrm{FN}$ rates are lower in patients who receive pegfilgrastim than in those who receive 11 days of daily G-CSF (von Minckwitz et al, 2008), which suggests that the common practice of giving daily G-CSF for 4-7 days may provide suboptimal prophylaxis.

\section{Risk assessment}

The management of chemotherapy-induced FN is gaining increasing attention as evidence accumulates to indicate that a proactive, preventive approach may improve the delivery of care and patient outcomes. Both chemotherapy and patient-related factors are important when assessing which chemotherapy regimen and supportive treatments to give to a particular patient.

The risks associated with both the prescribed chemotherapy regimen and patient factors (Figure 1) must be assessed before commencing chemotherapy and before delivering each subsequent cycle (Aapro et al, 2006).

The choice of chemotherapy is generally the main risk factor for $\mathrm{FN}$, and if two different regimens are believed to offer equal efficacy to the patient, the regimen with the lower risk of FN should be chosen. However, looking specifically at patients with breast cancer, it is well documented that taxane-containing regimens are significantly more effective than those without taxanes (Peto et al, 2007), at the cost of increased FN rates (Bria et al, 2005).

Several patient risk factors are associated with development of FN. There is evidence from well-designed trials showing that FN is 
more likely to develop in patients older than 65 years and in those with advanced disease, a previous history of FN and low performance status (Aapro et al, 2006). There is also evidence suggesting other FN risk factors, including female sex, haemoglobin below $12 \mathrm{~g} \mathrm{dl}^{-1}$, cardiovascular disease, renal disease and abnormal liver function tests, in particular raised bilirubin (Aapro et al, 2006; Lyman et al, 2007). Careful assessment of all the treatment and patient factors should be formulated, and the guidelines closely adhered to.

\section{Elderly patients}

Elderly patients account for only a small proportion of the population with early-stage breast cancer (prevalence approaching $7 \%$ among the over-70 s) (Louwman et al, 2007). This group is worth mentioning, however, as elderly patients present particular challenges, including reduced tolerance to chemotherapy (Wedding et al, 2007) and greater susceptibility to the development of FN (Lyman and Dale, 2003). Importantly, with careful patient selection, chemotherapy can be effective and tolerable in older as well as younger individuals.

It is important to note that chronological age, by itself, is not a reliable indicator of life expectancy, functional reserve or the risk of treatment complications (Wedding et al, 2007). Elderly patients require a comprehensive geriatric assessment, looking at function, comorbidities, nutritional status, cognition, emotional evaluation and socioeconomic issues (Wedding et al, 2007).

A systematic review of the literature has provided evidence to support the use of haematopoietic growth factors in elderly people, to reduce the risk of neutropenic events and the need for reduced DI (Lyman and Dale, 2003), but specific evidence for the benefits of primary prophylaxis of FN in elderly breast cancer patients is currently lacking. Further studies are needed.

\section{ROLE OF SECONDARY PROPHYLAXIS WITH G-CSF}

Patients with a high risk of FN (over 20\%) should be offered primary prophylaxis with G-CSF, in accordance with guidelines (Aapro et al, 2006; Smith et al, 2006; Segal et al, 2008). However, a predicted low risk (under 10\%) of FN is no guarantee that the side effect will not occur. Secondary prophylaxis during subsequent cycles is therefore an option if a low-risk patient develops FN during chemotherapy.

There are no prospective studies of the value of secondary G-CSF prophylaxis (Hupperets and Tjan-Heijnen, 2006). After patients in a pivotal G-CSF trial were allowed to switch from placebo to G-CSF if they developed FN in the first cycle (Crawford et al, 1991), the FN rate fell from $100 \%$ in cycle 1 to $23 \%$ in cycle 2 . However, definite conclusions on the efficacy of secondary prophylaxis cannot be drawn from these observations because many trials report a decline in the incidence of $\mathrm{FN}$ in later cycles without administering prophylaxis (Hupperets and Tjan-Heijnen, 2006).

Secondary prophylaxis should be offered if a patient develops $\mathrm{FN}$, despite being in a low FN risk category, and it is judged important to maintain their DI. However, dose reduction or delay may be appropriate for some patients such as, for example, those receiving palliative chemotherapy and those who develop grade $3 / 4$ non-haematological toxicities that cannot be attributable to FN. If a patient is likely to develop a serious complication resulting from $\mathrm{FN}$, such as death, then primary prophylaxis should be considered.

\section{CONCLUSION}

As well as being associated with morbidity and mortality (Aapro et al, 2006; Kuderer et al, 2006), chemotherapy-induced FN can also lead to treatment modifications and poorer outcomes for patients - a particular concern when chemotherapy is being given with curative intent (Aapro et al, 2006). However, primary prophylaxis of $\mathrm{FN}$, using G-CSF, can reduce the risk of FN developing in the first place (Aapro et al, 2006; Smith et al, 2006; Segal et al, 2008). Regimen-based and patient-based indications for its use are set out in international guidelines (Aapro et al, 2006; Smith et al, 2006; Segal et al, 2008). Patients whose risk of chemotherapy-induced FN is $20 \%$ or greater should be given primary prophylactic G-CSF. If a patient develops FN during a less intensive regimen, and DI needs to be maintained, secondary prophylactic G-CSF should be strongly considered for the support of subsequent treatment cycles.

\section{ACKNOWLEDGEMENTS}

Our thanks to Succinct Healthcare Communications and Consultancy for editorial support. This supplement is sponsored by an educational grant from sanofi-aventis. The company has checked the factual and medical content, but final editorial control resides with the author and editor.

\section{Conflict of interest}

$S$ Kelly has received lecture fees from sanofi-aventis and Amgen. D Wheatley has received advisory board fees from sanofi-aventis and lecture fees from Amgen.

\section{REFERENCES}

Aapro MS, Cameron DA, Pettengell R, Bohlius J, Crawford J, Ellis M, Kearney N, Lyman GH, Tjan-Heijnen VC, Walewski J, Weber DC, Zielinskil C, European Organisation for Research and Treatment of Cancer (EORTC) Granulocyte Colony-Stimulating Factor (G-CSF) Guidelines Working Party (2006) EORTC guidelines for the use of granulocyte-colony stimulating factor to reduce the incidence of chemotherapy-induced febrile neutropenia in adult patients with lymphomas and solid tumours. Eur J Cancer 42: 2433-2453

Bonadonna G, Valagussa P, Moliterni A, Zambetti M, Brambilla C (1995) Adjuvant cyclophosphamide, methotrexate, and fluorouracil in nodepositive breast cancer. $N$ Engl J Med 332: 901 - 906

Bonneterre J, Roché H, Kerbrat P, Brémond A, Fumoleau P, Namer M, Goudier MJ, Schraub S, Fargeot P, Chapelle-Marcillac I (2005) Epirubicin increases long-term survival in adjuvant chemotherapy of patients with poor-prognosis, node-positive, early breast cancer: 10-year follow-up results of the French Adjuvant Study Group 05 randomized trial. J Clin Oncol 23: $2686-2693$
Bria E, Giannarelli D, Felici A, Peters WP, Nisticò C, Vanni B, Cuppone F, Cognetti F, Terzoli E (2005) Taxanes with anthracyclines as first-line chemotherapy for metastatic breast carcinoma. Cancer 103: 672-679

Cameron D (2009) Management of chemotherapy-associated febrile neutropenia. Br J Cancer 101(Suppl 1): S18-S22

Chirivella I, Bermejo A, Insa A, Perez-Fidalgo A, Magro A, Rosello S, Garcia-Garre E, Martin P, Bosch A, Lluch A (2006) Impact of chemotherapy dose-related factors on survival in breast cancer patients treated with adjuvant anthracycline-based chemotherapy. J Clin Oncol 24: Abstract 668

Crawford J, Ozer H, Stoller R, Johnson D, Lyman G, Tabbara I, Kris M, Grous J, Picozzi V, Rausch G, Smith R, Gradishar W, Yahanda A, Vincent M, Stewart M, Glaspy J (1991) Reduction by granulocyte colonystimulating factor of fever and neutropenia induced by chemotherapy in patients with small cell lung cancer. N Engl J Med 325: 164-170

Cullen M, Baijal S (2009) Prevention of febrile neutropenia: use of prophylactic antibiotics. Br J Cancer 101(Suppl 1): S11-S14 
Gounaris I, Macleod P, Kelly S (2008) Feasibility of Adjuvant Treatment with Docetaxel/Doxorubicin/Cyclophosphamide (the TAC regime) in Routine Clinical Practice. Plymouth Hospitals NHS Trust: Plymouth

Herbst C, Naumann F, Kruse EB, Monsef I, Bohlius J, Schulz H, Engert A (2009) Prophylactic antibiotics or G-CSF for the prevention of infections and improvement of survival in cancer patients undergoing chemotherapy. Cochrane Database Syst Rev 21: CD007107

Hupperets PS, Tjan-Heijnen VC (2006) Primary or secondary G-CSF prophylaxis to support TAC chemotherapy in breast cancer? Ann Oncol 17: $1181-1183$

Komrokji RS, Lyman GH (2004) The colony stimulating factors: use to prevent and treat neutropenia and its complications. Expert Opin Bio Ther 4: $1897-1910$

Kuderer NM, Dale DC, Crawford J, Cosler LE, Lyman GH (2006) Mortality, morbidity, and cost associated with febrile neutropenia in adult cancer patients. Cancer 106: $2258-2266$

Kuderer NM, Dale DC, Crawford J, Lyman GH (2007) Impact of primary prophylaxis with granulocyte colony-stimulating factor on febrile neutropenia and mortality in adult cancer patients receiving chemotherapy: a systematic review. J Clin Oncol 25: 3158-3167

Leonard RCF, Miles D, Thomas R, Nussey F, UK Breast Cancer Neutropenia Audit Group (2003) Impact of neutropenia on delivering planned adjuvant chemotherapy: UK audit of primary breast cancer patients. $\mathrm{Br} J$ Cancer 89: $2062-2068$

Louwman WJ, Vulto JCM, Verhoeven RHA, Nieuwenhuijzen GAP, Coebergh JWW, Voogd AC (2007) Clinical epidemiology of breast cancer in the elderly. Eur J Cancer 43: 2242-2252

Lyman GH, Crawford J, Wolff DA, Culakova E, Poniewierski MS, Dale DC, for the ANC Study Group (2007) A risk model for first cycle febrile neutropenia in cancer patients receiving systemic chemotherapy. J Clin Oncol 23: Abstract: 8122

Lyman GH, Dale DJ (2003) Risk and timing of hospitalization for febrile neutropenia in patients receiving CHOP, CHOP-R, or CNOP chemotherapy for intermediate-grade non-Hodgkin's lymphoma. Cancer 98: $2402-2409$

Martin M, Pienkowski T, Mackey J, Pawlicki M, Guastalla JP, Weaver C, Tomiak E, Al-Tweigeri T, Chap L, Juhos E, Guevin R, Howell A, Fornander T, Hainsworth J, Coleman R, Vinholes J, Modiano M, Pinter T, Tang SC, Colwell B, Prady C, Provencher L, Walde D, RodriguezLescure A, Hugh J, Loret C, Rupin M, Blitz S, Jacobs P, Murawsky M, Riva A, Vogel C, Breast Cancer International Research Group 001 Investigators (2005) Adjuvant docetaxel for node-positive breast cancer. $N$ Engl J Med 352: 2302-2313
Oncologist Breast Cancer Study (2008) sanofi-aventis. Data on file

Ozer H, Armitage JO, Bennett CL, Crawford J, Demetri GD, Pizzo PA, Schiffer CA, Smith TJ, Somlo G, Wade JC, Wade JL, Winn RJ, Wozniak AJ, Somerfield MR, for the American Society of Clinical Oncology Growth Factors Expert Panel (2000) 2000 Update of recommendations for the use of haematopoietic colony stimulating factors: evidence based, clinical practice guidelines. J Clin Oncol 18: $3558-3585$

Peto R, on behalf of the Early Breast Cancer Trialists' Collaborative Group (EBCTCG) (2007) The worldwide Oxford overview: Updated (2005-2006) meta-analyses of trial results. San Antonio Breast Cancer Symposium

Segal BH, Freifeld AG, Baden LR, Brown AE, Casper C, Dubberke E, Gelfand M, Greene JN, Ison MG, Ito JI, Karp JE, Kaul DR, King E, Mackler E, Marcucci G, Montoya JG, Engemann AM, Rolston K, The AS (2008) Prevention and treatment of cancer-related infections. J Natl Compr Canc Netw 6: $122-174$

Smith TJ, Khatcheressian J, Lyman GH, Ozer H, Armitage JO, Balducci L, Bennett CL, Cantor SB, Crawford J, Cross SJ, Demetri G, Desch CE, Pizzo PA, Schiffer CA, Schwartzberg L, Somerfield MR, Somlo G, Wade JC, Wade JL, Winn RJ, Wozniak AJ, Wolff AC (2006) 2006 update of recommendations for the use of white blood cell growth factors: an evidence-based clinical practice guideline. J Clin Oncol 24: 3187-3205

Vogel CL, Wojtukiewicz MZ, Carroll RR, Tjulandin SA, Barajas-Figueroa LJ, Wiens BL, Neumann TA, Schwartzberg LS (2005) First and subsequent cycle use of pegfilgrastim prevents febrile neutropenia in patients with breast cancer: a multicenter, double-blind, placebocontrolled phase III study. J Clin Oncol 23: 1178-1184

von Minckwitz G, Kummel S, du Bois A, Eiermann W, Eidtmann H, Gerber B, Hilfrich J, Huober J, Costa SD, Jackisch C, Grasshoff ST, Vescia S, Skacel T, Loibl S, Mehta KM, Kaufmann M, German Breast Group (2008) Pegfilgrastim \pm ciprofloxacin for primary prophylaxis with TAC (docetaxel/doxorubicin/cyclophosphamide) chemotherapy for breast cancer. Results from the GEPARTRIO study. Ann Oncol 19: $292-298$

Wedding U, Pientka L, Hoffken K (2007) Quality-of-life in elderly patients with cancer: a short review. Eur J Cancer 43: 2203-2210

Wood WC, Budman DR, Korzun AH, Cooper MR, Younger J, Hart RD, Moore A, Ellerton JA, Norton L, Ferree CR, Colangelo Ballow A, Frei E, Henderson IC (1994) Dose and dose intensity of adjuvant chemotherapy for stage II, node-positive breast carcinoma. $N$ Engl J Med 330: $1253-1259$ 